Bull. Austral. Math. Soc.

Vol. 49 (1994) [139-149]

\title{
ON GENERALISED CONVEX NONSMOOTH FUNCTIONS
}

\section{DiNh THE LUC}

Some characterisations of generalised convex functions are established by means of Clarke's subdifferential and directional derivatives.

\section{INTRODUCTION}

Let $f$ be a function from a real topological vector space $X$ to the extended real line $\mathbb{R} \cup\{+\infty\}$. For every $x, y \in \operatorname{dom} f$ and every real number $\lambda$, denote

$$
d(x, y, \lambda):=\max \{f(x), f(y)\}-f(\lambda x+(1-\lambda) y) .
$$

The function $f$ is said to be

(i) quasiconvex if

$$
d(x, y, \lambda) \geqslant 0 \text { for every } x, y \in \operatorname{dom} f, \lambda \in(0,1) ;
$$

(ii) strictly quasiconvex if strict inequality holds in (1) when $x \neq y$;

(iii) semistrictly quasiconvex if strict inequality holds in (1) when $f(x) \neq f(y)$;

(iv) pseudoconvex if whenever $x, y \in \operatorname{dom} f$ with $f(y)>f(x)$, there exist $\beta(x, y)>0$ and $\delta(x, y) \in(0,1]$ such that

$$
d(x, y, \lambda) \geqslant \lambda \beta(x, y) \text { for every } \lambda \in(0, \delta(x, y)) \text {; }
$$

(v) strictly pseudoconvex if (2) holds whenever $f(y) \geqslant f(x), x \neq y$.

These and some other generalisations of convex functions have been investigated by numerous authors and they have been widely used in economics, operations research, engineering et cetera (see $[1,2,3,6,7,8,9,10,11,12,13,14,15,16,17,18,22]$ and the references given therein). Here we use the nonconventional definition of pseudoconvexity from [17] to avoid differentiability assumptions on the function. In [13] we introduced the concept of quasimonotone maps and proved that a lower semicontinuous function from a Banach space to the extended real line is quasiconvex if and only if its generalised subdifferential map is quasimonotone. The present paper is a continuation of [13]. Our purpose is to exploit generalised (Clarke's) subdifferential and directional derivatives to characterise the functions of types (ii) $-(v)$. The results to be proven are especially useful when dealing with nonsmooth functions. They extend at the same time several characterisations of generalised convex functions previousely established by other authors.

Received 30th March, 1993

The paper was partly written when the author was at the University of Erlangen-Nurnberg under a grant of the Alexander von Humboldt Foundation.

Copyright Clearance Centre, Inc. Serial-fee code: 0004-9729/94 \$A2.00+0.00. 


\section{SUBDifFERENTIAL OF A GENERALISED CONVEX FUNCTION}

Throughout this section we suppose that $X$ is a Banach space and $f$ is a lower semicontinuous function from $X$ to $\mathbb{R} \cup\{+\infty\}$. For a point $x \in \operatorname{dom} f$, the Clarke generalised subderivative of $f$ at $x$ in direction $v \in X$ is defined by

$$
f^{\dagger}(x ; v)=\sup _{e>0} \limsup _{(y, \alpha) \perp_{f} z ; \downarrow t 0} \inf _{u \in B(v, e)} \frac{f(y+t u)-\alpha}{t}
$$

where $(y, \alpha) \downarrow_{f} x$ means that $y \rightarrow x, \alpha \rightarrow f(x), \alpha \geqslant f(y)$, and $B(v, \varepsilon)$ is the ball in $X$ with centre at $v$ and radius $\varepsilon$ (see $[4,19,20]$ ). If $f$ is Lipschitz around $x$ the above formula takes a simple form:

$$
f^{\dagger}(x ; v)=\limsup _{y \rightarrow x ; t ! 0} \frac{f(y+t v)-f(y)}{t} .
$$

The generalised (Clarke's) subdifferential of $f$ at $x$ is

$$
\partial f(x)=\left\{x^{*} \in X^{\prime}:\left\langle x^{*}, v\right\rangle \leqslant f^{\dagger}(x ; v) \text { for all } v \in X\right\},
$$

where $X^{\prime}$ is the topological dual space of $X$, and $\langle.,$.$\rangle is the pairing between X$ and $X^{\prime}$. If $x \notin \operatorname{dom} F$, one sets $\partial f(x)=\emptyset$.

We recall that a set valued map $F$ from $X$ to its topological dual $X^{\prime}$ is said to be monotone (respectively, quasimonotone) if for every $x, y \in X$ with $x \neq y$, and for every $x^{*} \in F(x), y^{*} \in F(y)$ one has

$$
\left\langle x^{*}, y-x\right\rangle+\left\langle y^{*}, x-y\right\rangle \leqslant 0,
$$

(respectively, $\min \left\{\left\langle x^{*}, y-x\right\rangle,\left\langle y^{*}, x-y\right\rangle\right\} \leqslant 0$ ).

If the above inequality is strict, one says that $F$ is strictly monotone (respectively, strictly quasimonotone). As in [13] it can be shown that when $F$ is a linear operator from a Hilbert space to itself, the following properties are equivalent:

(a) $F$ is strictly monotone,

(b) $F$ is strictly quasimonotone,

(c) the symmetric part of $F$ is positive definite.

It was proven in $[12,13]$ that $f$ is convex (respectively, quasiconvex) if and only if $\partial f$ is monotone (respectively, quasimonotone) (see also [5] for convex functions and [7] for quasiconvex Lipschitz functions). For strictly convex functions we have the following result. 
THEOREM 2.1. If $f$ is strictly convex, then $\partial f$ is strictly monotone. Conversely, if $\partial f$ is strictly monotone and nonvoid, then $f$ is strictly convex.

Proof: Suppose that $f$ is strictly convex. If $\partial f$ is not strictly monotone, there must exist $x, y \in X$ with $x \neq y$ and $x^{*} \in \partial f(x), y^{*} \in \partial f(y)$ such that

$$
\left\langle x^{*}, y-x\right\rangle+\left\langle y^{*}, x-y\right\rangle \geqslant 0 .
$$

On the other hand, by the convexity one has

$$
\begin{aligned}
& f\left(\frac{x+y}{2}\right)-f(x) \geqslant\left\langle x^{*}, \frac{y-x}{2}\right\rangle, \\
& f\left(\frac{x+y}{2}\right)-f(y) \geqslant\left\langle y^{*}, \frac{x-y}{2}\right\rangle .
\end{aligned}
$$

These inequalities and (3) imply that

$$
f\left(\frac{x+y}{2}\right) \geqslant \frac{1}{2}(f(x)+f(y))
$$

which contradicts the strict convexity of $f$. For the converse part, observe first that since $\partial f(x)$ is nonempty for every $x \in X$, the function $f$ is everywhere finite, and since $\partial f$ is monotone, by Theorem 3.1 of [12], $f$ is convex. Hence it is Lipschitz near every compact subset of the space. Now suppose that $f$ is not strictly convex, that is, there can be found $a, b \in X, a \neq b$ and $c=\lambda a+(1-\lambda) b$, some $\lambda \in(0,1)$, such that

$$
f(c) \geqslant \lambda f(a)+(1-\lambda) f(b) .
$$

By Lebourg's mean value theorem [4], there exist $x \in(a, c), y \in(c, b)$ and $x^{*} \in$ $\partial f(x), y^{*} \in \partial f(y)$ such that

$$
\begin{aligned}
& f(c)-f(a)=\left\langle x^{*}, c-a\right\rangle, \\
& f(c)-f(b)=\left\langle y^{*}, c-b\right\rangle .
\end{aligned}
$$

With these equalities in hand we calculate the sum

$$
\begin{aligned}
\left\langle x^{*}, y-x\right\rangle+\left\langle y^{*}\right. & , x-y\rangle \\
& =\frac{\|y-x\|}{\|c-a\|}\left\langle x^{*}, c-a\right\rangle+\frac{\|y-x\|}{\|c-b\|}\left\langle y^{*}, c-b\right\rangle \\
& =\|y-x\|\left\{\frac{f(c)-f(a)}{(1-\lambda)\|b-a\|}+\frac{f(c)-f(b)}{\lambda\|a-b\|}\right\} \\
& =\frac{\|y-x\|}{\|b-a\|} \frac{1}{\lambda(1-\lambda)}\{\lambda f(c)-\lambda f(a)+(1-\lambda) f(c)-(1-\lambda) f(b)\} \\
& \geqslant 0 .
\end{aligned}
$$


This shows that $\partial f$ is not strictly monotone and the proof is complete.

The proof of the above theorem also reveals that the nonemptiness of the subdifferential can be replaced by the finiteness of $f(x)$. The strict monotonicity of $\partial f$ alone is not sufficient for the strict convexity. For instance, the function of two variables $f(x, y)$ from $\mathbb{R}^{2}$ to $\mathbb{R} \cup\{+\infty\}$ is defined by the rule

$$
f(x, y)= \begin{cases}\left(\sqrt{1-y^{2}}+1\right)\left(x^{2}-1\right) & \text { if }|x| \leqslant 1,|y| \leqslant 1 \\ +\infty & \text { otherwise }\end{cases}
$$

has a strictly monotone subdifferential (by a direct calculation). However, it is not strictly convex, for it is constant on the interval $x=1,|y| \leqslant 1$. Furthermore, the second part of Theorem 2.1 remains true if we restrict the function on an open convex subset of the space. The proof goes through without change.

THEOREM 2.2. Assume that $f$ is Lipschitz on an open convex set and $\partial f$ is strictly quasimonotone. Then $f$ is strictly pseudoconvex on this set.

Proof: Suppose to the contrary that $f$ is not strictly pseudoconvex, that is, there exist two points $a, b, a \neq b$ of the open convex set with $f(b) \geqslant f(a)$ such that for every positive numbers $\varepsilon$ and $\delta \leqslant 1$ one can find $\lambda \in(0, \delta]$ satisfying inequality

$$
f(\lambda a+(1-\lambda) b)>f(b)-\lambda \varepsilon .
$$

Let $c \in[a, b]$ be a point which minimises $f(x)$ on $[a, b]$. It is clear that $c \neq b$ because otherwise $f(x)$ would be constant on $[a, b]$ and $\partial f$ would not be strictly quasimonotone. By Lebourg's mean value theorem [4] there exist a point $x \in(c, b)$ and $x^{*} \in \partial f(x)$ such that

$$
\left\langle x^{*}, b-c\right\rangle=f(b)-f(c)
$$

Hence

$$
\begin{aligned}
\left\langle x^{*}, b-x\right\rangle & =\frac{\|b-x\|}{\|b-c\|}\left\langle x^{*}, b-c\right\rangle \\
& =\frac{\|b-x\|}{\|b-c\|}(f(b)-f(c)) \\
& >0 .
\end{aligned}
$$

Consider now the Clarke directional derivative of $f$ at $b$ in direction $a-b$ :

$$
\begin{aligned}
f^{0}(b, a-b) & =\limsup _{y \rightarrow b, t \downarrow 0} \frac{f(y+t(a-b))-f(y)}{t} \\
& \geqslant \limsup _{t \downarrow 0} \frac{f(b+t(a-b))-f(b)}{t} .
\end{aligned}
$$


In view of (4) and since $\varepsilon$ can be chosen arbitrarily, one concludes that $f^{0}(b, a-b) \geqslant 0$. By the Lipschitz condition,

$$
f^{0}(b, a-b)=\max _{y^{*} \in \theta f(b)}\left\langle y^{*}, a-b\right\rangle
$$

This means that there must be some $y^{*} \in \partial f(b)$ such that

$$
\begin{aligned}
\left\langle y^{*}, a-b\right\rangle & =f^{0}(b, a-b) \geqslant 0 . \\
\text { Hence, } & \left\langle y^{*}, x-b\right\rangle=\frac{\|x-b\|}{\|a-b\|}\left\langle y^{*}, a-b\right\rangle \geqslant 0 .
\end{aligned}
$$

The latter inequality and (5) contradict the strict quasimonotonicity of $\partial f$. The proof is complete.

The function given after Theorem 2.1 has a strictly monotone, hence strictly quasimonotone subdifferential, however it is neither strictly pseudoconvex, nor strictly quasiconvex. The converse of Theorem 2.2 is in general not true. For instance, the function $f$ from $\mathbb{R}$ to $\mathbb{R}$ defined by the rule

$$
f(x)= \begin{cases}x & \text { if } x \leqslant 0 \\ x^{2} & \text { otherwise }\end{cases}
$$

is strictly pseudoconvex, however at $x=-1, y=0$ one has $\partial f(x)=\{1\}, \partial f(y)=$ $[0,1]$. Hence $\partial f$ is not strictly quasimonotone. (The function is of course Lipschitz on any open bounded subset of $\mathbb{R}$.) To formulate further results we shall make use of the following terminology: we say that $\partial f$ is semistrictly quasimonotone on a set $A \subseteq X$ if for every $x, y \in A$ with $f(x) \neq f(y)$, for every $x^{*} \in \partial f(x), y^{*} \in \partial f(y)$ one has

$$
\min \left\{\left\langle x^{*}, y-x\right\rangle,\left\langle y^{*}, x-y\right\rangle\right\}<0 .
$$

THEOREM 2.3. Assume that $f$ is Lipschitz on an open convex set and its subdifferential is semistrictly quasimonotone there. Then $f$ is pseudoconvex (hence semistrictly quasiconvex as well) on this set.

Proof: Suppose to the contrary that $f$ is not pseudoconvex, that is, there exist two points $a, b$ on the open convex set with $f(b)>f(a)$ such that for every positive number $\varepsilon$ and $\delta \leqslant 1$, one can find $\lambda \in(0, \delta]$ such that

$$
f(\lambda a+(1-\lambda) b)>f(b)-\lambda \varepsilon .
$$

Take a point $c \in(a, b)$ with the property that

$$
f(a)<f(c), f(x)<f(b) \text { for every } x \in[a, c] .
$$


Such a point exists because $f$ is continuous (on the set we are considering) and $f(a)<$ $f(b)$. Apply Lebourg's mean value theorem to $f$ on $[a, c]$ to get point $x \in(a, c)$ and $x^{*} \in \partial f(x)$ such that $\left\langle x^{*}, c-a\right\rangle=f(c)-f(a)$. By $(7), f(x)<f(b)$ and

$$
\begin{aligned}
\left\langle x^{*}, b-x\right\rangle & =\frac{\|b-x\|}{\|c-a\|}\left(x^{*}, c-a\right\rangle \\
& =\frac{\|b-x\|}{\|c-a\|}(f(c)-f(a)) \\
& >0
\end{aligned}
$$

Using (6) and the argument of the proof of Theorem 2.2 one can show that $\left\langle b^{*}, a-b\right\rangle \geqslant 0$ for some $b^{*} \in \partial f(b)$. This implies also that $\left\langle b^{*}, x-b\right\rangle \geqslant 0$, which together with (8) contradicts the semistrict quasimonotonicity of $f$.

Observe that the condition stated in Theorem 2.3 is merely sufficient for the semistrict pseudoconvexity. The example given after Theorem 2.2 shows that a pseudoconvex Lipschitz function need not fulfill it.

\section{Directional Derivatives}

In this section we suppose that $X$ is a real topological vector space and $f$ is a lower semicontinuous function from $X$ to $\mathbb{R} \cup\{+\infty\}$. We recall that the directional upper and lower (Dini) derivatives of $f$ at $x \in \operatorname{dom} f$ are the maps $f_{+}^{\prime}(x,$.$) and f_{-}^{\prime}(x,$.$) from$ $X$ to $\mathbb{R} \cup\{ \pm \infty\}$ defined by

and

$$
\begin{aligned}
& f_{+}^{\prime}(x, v)=\limsup _{t \downarrow 0} \frac{f(x+t v)-f(x)}{t} \\
& f_{-}^{\prime}(x, v)=\liminf _{t \downarrow 0} \frac{f(x+t v)-f(x)}{t}
\end{aligned}
$$

for every $v \in X$. In the case the two above limits are equal, it is called the directional derivative of $f$ at $x$ in direction $v$ and denoted by $f^{\prime}(x, v)$. If $x \notin \operatorname{dom} f$, we set $f^{\prime}(x, v)=-\infty$ for every $v \in X$. We recall also that a bifunction $h$ from $X$ to $\mathbb{R} \cup\{ \pm \infty\}$, (that is, a function from the product space $X \times X$ to $\mathbb{R} \cup\{ \pm \infty\}$ ) is said to be monotone (respectively, quasimonotone) if for every $x, y \in X, x \neq y$ one has

$$
\begin{gathered}
h(x, y-x)+h(y, x-y) \leqslant 0, \\
\text { (respectively, } \min \{h(x, y-x), h(y, x-y)\} \leqslant 0) .
\end{gathered}
$$

If the above inequalities are strict, we say that $h$ is strictly. monotone (respectively, strictly quasimonotone). Similar to the concept of monotone maps of the previous 
section, the strict monotonicity, the strict quasimonotonicity of a linear bifunction and the positive definiteness of its symmetric part are all equivalent. In [11] it was proven that $f(x)$ is strictly convex if and only if the bifunction $f_{+}^{\prime}$ is strictly monotone. It is not difficult to show that the same is true for the bifunction $f_{-}^{\prime}$. For quasiconvex functions we established in [13] that $f(x)$ is quasiconvex if and only if the bifunction $f_{-}^{\prime}$ (or $f_{+}^{\prime}$ ) is quasimonotone. Let us now study the functions of types (ii)-(v) described in the introduction. We shall make use of the following result from [13] which we denote by (MVT) (the abbreviation of "mean value theorem"): for every $a, b \in X$ with $f(a)$ finite there exists a point $c \in[a, b)$ such that $f_{-}^{\prime}(c, b-a) \geqslant f_{0}$, where $f_{0}$ denotes $f(b)-f(a)$ if $f(b)$ is finite and any fixed positive number if $f(b)=+\infty$.

THEOREM 3.1. The function $f$ is strictly quasiconvex if and only if the bifunction $f_{-}^{\prime}$ is quasimonotone and for every fixed $x, v \in X, v \neq 0$ the set $\{y \in[x, x+v]$ : $\left.f_{-}^{\prime}(y, v) \neq 0\right\}$ is dense in $[x, x+v]$.

Proof: Assume that $f$ is strictly quasiconvex. In particular it is quasiconvex, hence $f_{-}^{\prime}$ is quasimonotone by Theorem 5.2 of [13]. Suppose that the density condition does not hold, that is, there are $x, v \in X, v \neq 0$ and a positive $\varepsilon$ such that

$$
f_{-}^{\prime}(y, v)=0 \quad \text { for every } y \in[x, x+\varepsilon v] .
$$

Consider the function $f$ on $[x, x+\varepsilon v]$. By (MVT), as a function of the variable $t$ the function $f(x+t v)$ is nonincreasing on $[0, \varepsilon)$. It follows from (9) that for any fixed positive $\delta$ one has $f(x+t v) \leqslant f(x)+t \delta$ for every $t \in[0, \varepsilon)$. This means that $f(x+t v)$ is constant on $[0, \varepsilon)$, which contradicts the strict quasiconvexity assumption. Conversely, if $f_{-}^{\prime}$ is quasimonotone, in view of Theorem 5.2 of $[13], f$ is quasiconvex. If it is not strictly quasiconvex, there exist $a, b \in \operatorname{dom} f, c \in(a, b)$ with $f(b) \geqslant f(a)$ such that $f(c)=f(b) \geqslant f(a)$. Consider the case $f(b)>f(a)$ first. By the quasiconvexity,

$$
f(y)=f(c) \quad \text { for every } y \in[c, b] .
$$

Taking $x=c, v=b-c$ we see that $f_{-}^{\prime}(y, v)=0$ for every $y \in[x, x+v)$, which contradicts the density condition. Thus, the case $f(b)>f(a)$ is impossible and must have $f(b)=f(a)$. If there is some $y \in(c, b)$ such that $f(y)<f(b)$, then by the quasiconvexity one has that $f(x)=f(a)$ for every $x \in[a, c]$ and we arrive at a similar contradiction as above. It not, $f(y)=f(b)$ for all $y \in[c, b]$. We return to the situation (10) and the proof is complete.

For continuous functions the following fact strengthens the result of [6] which dealt with differentiable functions.

Proposition 3.2. Assume that $f$ is continuous on an open convex set $C \subseteq$ $X$. Then it is strictly quasiconvex on $C$ if and only if whenever $f_{-}^{\prime}(x, \pm v) \leqslant 0$ for 
some $x \in C, v \in X, v \neq 0$, as a function of $t, f(x+t v)$ cannot attain local maximum at $t=0$.

Proof: The "only if" part is obvious. It is true even without the condition on the directional derivative at $x$. For the "if" part, suppose to the contrary that the function is not strictly quasiconvex on $C$. One can find $a, b \in C, c \in(a, b)$ such that $f(c) \geqslant \max \{f(a), f(b)\}$. Since $f$ is continuous, it attains a maximum at some point $x$ on $[a, b]$. We may assume that $x \in(a, b)$. Take $v=b-a$ to have a contradiction to the assumption of the proposition.

THEOREM 3.3. The function $f$ is semistrictly quasiconvex if and only if the bifunction $f_{-}^{\prime}$ is quasimonotone and for every fixed $x, v \in X, v \neq 0$ with $f(x)>$ $f(x+v)$ there exists a positive $\varepsilon$ such that the set $\left\{y \in[x, x+\varepsilon v]: f_{-}^{\prime}(y, v) \neq 0\right\}$ is dense in $[x, x+\varepsilon v]$.

Proof: Assume that $f$ is semistrictly quasiconvex. In particular it is quasiconvex (see [9]). By Theorem 5.2 of [13], $f_{-}^{\prime}$ is quasimonotone. If the density condition does not hold, one can find an interval inside $[x, x+\varepsilon v]$ on which the directional lower derivatives of $f$ in direction $v$ are zero. This implies that $f$ is constant on that interval. When $\varepsilon$ is small enough, it follows from the lower semicontinuity of $f$ that the constant value must be greater then $f(x+v)$. Hence $f$ cannot be semistrictly quasiconvex. Conversely, if $f_{-}^{\prime}$ is quasimonotone, by Theorem 5.2 of [13] the function is quasiconvex. Suppose that it is not semistrictly quasiconvex, that is, there are $a, b \in X, c \in(a, b)$ such that $f(c)>f(a)=f(b)$. Take a positive $\varepsilon$ so small that $f(x)>f(b)$ for every $x \in[c, c+\varepsilon(b-c)]$. By the quasiconvexity we have that $f(x)=f(c)$ for all $x \in[c, c+\varepsilon(b-c)]$. This contradicts the density condition of the theorem.

As in the previous section, we shall say that the bifunction $f_{-}^{\prime}$ is semistrictly quasimonotone if whenever $x, y \in X$ with $f(x) \neq f(y)$, one has

$$
\min \left\{f_{-}^{\prime}(x, y-x), f_{-}^{\prime}(y, x-y)\right\}<0 .
$$

THEOREM 3.4. $f$ is strictly pseudoconvex (respectively, pseudoconvex) if and only if $f_{-}^{\prime}$ is strictly quasimonotone (respectively, semistrictly quasimonotone).

Proof: Assume that $f$ is strictly pseudoconvex and $x, y \in X, x \neq y$ with $f(y) \geqslant$ $f(x)$. If $f(y)=\infty$, by the definition $f_{-}^{\prime}(y, x-y)=-\infty$. We may therefore assume that $f(y)$ is finite. By the strict pseudoconvexity,

$$
\frac{f(y+\lambda(x-y))-f(y)}{\lambda}<-\beta(x, y)
$$

for every $\lambda \in(0, \delta(x, y))$, some $\beta(x, y)>0, \delta(x, y) \in(0,1]$. Consequently $f_{-}^{\prime}(y, x-y) \leqslant$ $-\beta(x, y)$ and indeed $f_{-}^{\prime}$ is strictly quasimonotone. Conversely, if $f$ is not strictly 
pseudo-convex, there are $x, y \in X, x \neq y$ such that for every $\beta>0, \delta \in(0,1]$ one has

$$
f(\lambda x+(1-\lambda) y)>f(y)-\lambda \beta,
$$

for some $\lambda \in(0, \delta)$. We state that

$$
f_{-}^{\prime}(y, x-y) \geqslant 0 .
$$

In fact, if not, say $f_{-}^{\prime}(y, x-y)<-2 \varepsilon$ for some positive $\varepsilon$, then $f(\lambda x+(1-\lambda) y)-$ $f(y)<-\lambda \varepsilon$ whenever $\lambda$ is close to 0 . This contradicts (11) if we take $\beta=\varepsilon$. Furthermore, since $f(y) \geqslant f(x)$, in view of (MVT) there exists some $z \in[x, y)$ such that $f_{-}^{\prime}(z, y-x) \geqslant f(y)-f(x) \geqslant 0$. The latter inequality and (12) show that $f_{-}^{\prime}(z, y-z) \geqslant 0, f_{-}^{\prime}(y, z-y) \geqslant 0$. Hence $f_{-}^{\prime}$ is not strictly quasimonotone. For the pseudoconvexity the proof is similar.

It is enough to know that $f_{-}^{\prime}(x, v)$ is positively homogeneous in the second variable. If it happens to be homogeneous in this variable, for instance when $f$ is differentiable, the following strengthened version of a result of [6] can be derived from Theorem 3.4.

Corollary 3.5. Assume that $f$ is continuous and $f_{-}^{\prime}$ is homogeneous in the second variable on an open convex set $C \subseteq X$. Then $f$ is (strictly) pseudoconvex on $C$ if and only if $f_{-}^{\prime}(x, v)=0$ for some $x \in C, v \neq 0$ implies that as a function of the variable $t, f(x+t v)$ attains a (strict) local minimum at $t=0$.

Proof: Assume that $f$ is pseudoconvex on $C$. In view of Theorem 3.4, $f_{-}^{\prime}$ is semistrictly quasimonotone. Suppose further that $f_{-}^{\prime}(x, v)=0$, for some $x \in C, v \neq$ 0 . If $f(x+t v)<f(x)$ for some small $t$, then by (MVT) there exists a point $y \in$ $[x+t v, x)$ such that $f_{-}^{\prime}(y,-t v) \geqslant f(x)-f(x+t v)>0$. This and the fact $f_{-}^{\prime}(x, \pm v)$ $=0$ contradict the semistrict quasimonotonicity of $f_{-}^{\prime}$. Hence $f(x+t v)$ must attain a local minimum at $t=0$. Conversely, if $f$ is not pseudoconvex, by Theorem 3.4 there are $x, y \in C$ with $f(y)>f(x)$ such that $f_{-}^{\prime}(x, y-x) \geqslant 0, f_{-}^{\prime}(y, x-y) \geqslant 0$. Let $c \in[a, b]$ be a point maximising $f$ over $[a, b]$. It follows from the condition of the corollary that $c=y$ and $f_{-}^{\prime}(y, x-y)=0$. But this again contradicts the above mentioned condition. Thus $f$ must be pseudoconvex. The proof of the strict pseudoconvex case is similar.

Another interesting corollary is a result of Schaible [21] concerning quadratic functions.

COROLlaRY 3.6. Let $f(x)=(x A x / 2)+b x$ be a quadratic function on $\mathbb{R}^{n}$, where $A$ is a symmetric $n \times n$-matrix and $b$ is an $n$-vector. Then it is quasiconvex on an open convex set $C \subseteq \mathbb{R}^{n}$ if and only if it is pseudoconvex on this set.

Proof: The "if" part is obvious. Assume that $f$ is quasiconvex on $C$. By Theorem 5.2 of [13], for every $x, y \in C$ one has

$$
\min \{\langle A x+b, y-x\rangle,\langle A y+b, x-y\rangle\} \leqslant 0 .
$$


For two arbitrary points $x_{0}, y_{0} \in C$

$$
\frac{1}{2} y_{0} A y_{0}+b y_{0}>\frac{1}{2} x_{0} A x_{0}+b x_{0}
$$

we have to show that the inequality in (13) is strict. Set $l(x, y)=\langle A y+b, x-y\rangle$. Note that $l(x, y)$ is continuous in both variables and linear in the first. Let us suppose to the contrary that strict inequality in (13) does not hold, that is, $l\left(x_{0}, y_{0}\right) \geqslant 0$ and $l\left(y_{0}, x_{0}\right) \geqslant$ 0 . In view of (13), it suffices to consider two cases: (I) $l\left(x_{0}, y_{0}\right)=0, l\left(y_{0}, x_{0}\right)=0$ and (II) $l\left(x_{0}, y_{0}\right)=0, l\left(y_{0}, x_{0}\right)>0$. The first case is impossible because it implies that the values of $f$ at $x_{0}$ and $y_{0}$ are equal, which contradicts (14). We treat now the second case. If $l\left(x, y_{0}\right)$ is not identically zero in a neighbourhood of $x_{0}$, then we can choose $x \in C$ as near to $x_{0}$ as we want so that $l\left(x, y_{0}\right)>0$. By the continuity of $l$, for this point one still has $l\left(y_{0}, x\right)>0$. The two latter inequalities contradict (13). It follows from (14) that $l(x, y)$ cannot be identically zero in a neighbourhood of $\left(x_{0}, y_{0}\right)$ in $\mathbb{R}^{n} \times \mathbb{R}^{n}$. Hence for every neighbourhoods $U$ of $x_{0}$ and $V$ of $y_{0}$ in $\mathbb{R}^{n}$, there exists $x \in U, y \in V$ such that $l(x, y) \neq 0$. Again by the continuity of $l$ one may assume that $l\left(y^{\prime}, x^{\prime}\right)>0$ for every $x^{\prime} \in U, y^{\prime} \in V$. Taking $y$ in the role of $y_{0}$ we return to the first situation of the second case. Thus both cases (I), (II) are impossible, and the inequality in (13) for $x_{0}, y_{0}$ must be strict. By Theorem 3.4, $f$ is pseudoconvex.

We close this paper with the remark that the results presented in the last section remain true if we use directional upper derivatives instead of directional lower derivatives. The proofs are the same, noting that (MVT) is valid also for directional upper derivatives.

\section{REFERENCES}

[1] M. Avriel, W.E. Diewert, S. Schaible and I. Zang, Generalized concavity (Plenum Press, New York and London, 1988).

[2] K.J. Arrow and A.C. Enthoven, 'Quasiconcave programming', Econometrica 29 (1961), 779-800.

[3] A. Cambini, E. Castagnoli, L. Martein, P. Mazzoleni and S. Schaible (eds), Generalized convexity and fractional programming with economic applications, Lecture Notes in Economics and Mathematical Systems 345 (Springer-Verlag, Berlin, Heidelberg, New York, 1990).

[4] F.H. Clarke, Optimization and nonsmooth analysis (Wiley, New York, 1983).

[5] R. Correa, A. Jofre and L. Thibault, 'Characterization of lower semicontinuous convex functions', Proc. Amer. Math. Soc. 116 (1992), 67-72.

[6] W.E. Diewert, M. Avriel and I. Zang, 'Nine kinds of quasiconcavity and concavity', J. Econom. Theory 25 (1981), 397-420.

[7] R. Ellaia and H. Hassouni, 'Characterizations of nonsmooth functions through their generalized gradients', Optimization 22 (1991), 401-416. 
[8] J.-B. Hiriart-Urruty, 'Miscellanies on nonsmooth analysis and optimization', in Lecture Notes in Economics and Mathematics Systems 255 (Springer-Verlag, Berlin, Heidelberg, New York, 1986), pp. 8-24.

[9] S. Karamardian, 'Duality in mathematical programming', J. Math. Anal. Appl. 20 (1967), 344-358.

[10] S. Komlosi, 'Some properties on nondifferentiable pseudoconvex functions', Math. Programming 26 (1983), 232-237.

[11] D.T. Luc and S. Swaminathan, 'A characterization of convex functions', J. Nonlinear Analysis Theory, Methods and Appl. (to appear).

[12] D.T. Luc, 'On the maximal monotonicity of subdifferentials', Acta Math. Vietnam 18 (1993), 99-106.

[13] D.T. Luc, 'Characterizations of quasiconvex functions', Bull. Austral. Math. Soc. 48 (1993), 393-405.

[14] O.L. Mangasarian, Nonlinear programming (McGraw-Hill Book Co., New York, N.Y., 1969).

[15] J.-E. Martinez-Legaz, 'Weak lower subdifferentials and applications', Optimization 21 (1990), 321-341.

[16] P. Mazzoleni (ed.), Generalized concavity for economic applications (Pisa University, Pisa, 1992).

[17] J.M. Ortega and W.C. Rheinboldt, Iterative solution of nonlinear equations in several variables (Academic Press, New York and London, 1970).

[18] J. Ponstein, 'Seven kinds of convexity', Siam Review 9 (1967), 115-119.

[19] R.T. Rockafellar, 'Generalized dirivatives and subgradients of nonconvex functions', Canad. J. Math. 32 (1980), 257-280.

[20] R.T. Rockafellar, The theory of subgradients and its applications to problems of optimization. Convex and nonconvex functions (Heldermann Verlag, Berlin, 1981).

[21] S. Schailbe, 'Generalized convexity of quadratic functions', in Generalized concavity in optimization and economics, (S. Schaible and W.T. Ziemba, Editors) (Academic Press, New York, 1981), pp. 183-197.

[22] S. Schaible and W.T. Ziemba, Generalized concavity in optimization and economics (Academic Press, New York, 1981).

Institute of Mathematics

PO Box 631 Boho

Hanoi

Vietnam 